\author{
Kristina Lahl
}

\title{
Angst- und Sehnsuchtsräume. Repräsentationen der Natur in Computerspielen
}

\author{
Schlüsselwörter: Computerspiele, Natur, Narrative, Kultivierung, \\ Handlungsmacht
}

DOI: 10.4312/ars.13.2.285-299

Games Studies sind noch ein relativ junger eigenständiger Zweig der interdisziplinären Medienwissenschaft und mussten die Anerkennung der wissenschaftlichen und kulturellen Relevanz ihres Untersuchungsgegenstands hart erkämpfen. ${ }^{1}$ Dabei war es vor allen Dingen ein Aspekt, der in der Forschung das Alleinstellungsmerkmal des Mediums Computerspiel im Gegensatz z. B. zu Literatur und Film markiert: "[S]pace is the one category that has come to be accepted as the central issue of game studies, and the one in which all previous categories are integrated" (Günzel, 2008, 171). Die hochkomplexe Verknüpfung von Rezeption, Interaktion und Produktion, die Computerspielen inhärent ist und sich in ihrem Verhältnis von Spiel, Spielerin und Avatar oder Interface äußert, kommt insbesondere in der Repräsentation des Raums, der Aktion innerhalb desselben und der Re-aktion der Spielumgebung zur Geltung. Es liegt also nahe, eine Auseinandersetzung mit dem Raum als soziale und kulturelle Größe mit dem Computerspiel an das Medium zu knüpfen, das dessen Darstellungen als "raison d'être" innehat und auf vielfältige Weise nutzt (Aarseth, 2001, 309).

Raumdarstellungen in Computerspielen sind äußerst vielseitig und haben sich in der Historie des Genres sowohl mit der immerwährenden Erweiterung der technischen und graphischen Möglichkeiten als auch mit der Entstehung von verschiedenen Gameplay-Konzepten und darüber hinaus mit sich wandelnden kulturellen und sozialen Erwartungshorizonten weiterentwickelt. ${ }^{2}$ Der hochaktuelle Forschungszweig

1 Vgl. zum Überblick über die Forschung Freyermuth (2015).

2 Dabei geht der Begriff des Raums weit über eine visuelle Repräsentation am Bildschirm hinaus - in den Fokus rückt die Interaktion der Spielerin mit dem Raum, das Verhältnis zwischen Spielerin, Avatar und Raum, die Perspektiven in First Person- und Third Person-Games, die Differenzen zwischen dargestelltem und navigierbarem Raum, die soziale und kulturelle Gestaltung des Raums u. v. a. m. (vgl. z. B. Günzel, 2008). 
der Green Computer and Video Games, der sich erst mit der Publikation der Dissertation von Alenda Chang (2013) etabliert hat, nimmt wiederum einen besonderen Aspekt der Raumdarstellung in den Blick: die Repräsentation und Agency ${ }^{3}$ der Natur, wobei im Rahmen der aktuellen Entwicklungen und Debatten insbesondere das Klima im Fokus steht, wie auch die Sonderausgabe der Zeitschrift für Computerspielforschung Paidia mit dem Titel Repräsentationen und Funktionen von Umwelt im Computerspiel vom Februar 2018 beweist.

In diesem Beitrag soll anhand verschiedener Computerspiele mit TopDown- (insbesondere in Wirtschaftssimulationen) und Bottom-Up-Perspektive (insbesondere in Open World-Spielen) untersucht werden, welche Formen der Repräsentation der Natur in Computerspiele Eingang finden und welche Narrative zur Mensch-Natur-Interaktion dabei aufgegriffen und fortgeschrieben werden. Insbesondere steht dabei die These im Fokus, dass die Natur in den meisten Mainstream-Spielen lediglich eine Projektionsfläche für Machtphantasien des Menschen darstellt, der durch Technisierung oder Kultivierung die Natur von einem Angst- zu einem Sehnsuchtsraum umwandelt.

\section{II}

Die Umwelt (als environment im weiteren Sinne) kann unterschiedliche Funktionen innerhalb von Computerspielen einnehmen; Abraham und Jayemanne (2017) haben in Anlehnung an vorangehende Forschung vier verschiedene Modi der MenschUmwelt-Interaktion herausgearbeitet, wobei sie explizit darauf verweisen, dass diese sich nicht gegenseitig ausschließen, sondern ganz im Gegenteil parallel existieren oder jederzeit von einem Modus in den anderen übergehen können: Umwelt als Kulisse, als Ressource, als Gegner und als Text. Die Spielerin nimmt dabei in der Interaktion mit der simulierten Welt eine bestimmte Rolle ein, indem sie diese entdeckt, bezwingt, managt, kultiviert oder schlicht genießt. Das Verhältnis von Umwelt und Spielerin ist dabei stark abhängig vom Spielmodus, indem durch unterschiedliche Perspektiven und Erlebnismodi Macht- und Hierarchieverhältnisse zwischen den potentiellen Handlungsträgern determiniert werden (Möring, Schneider, 2018). So vermittelt der in Wirtschaftssimulationen übliche globale Panoramablick eines "gottähnlichen", kybernetischen Managers, der an allen Stellgrößen bzw. Einstellungen drehen kann, einen Top-Down-Ansatz, während Open-World-Spiele durch die ebenerdige und ins Spielgeschehen integrierte Perspektive des Avatars einen gleichberechtigteren Ansatz zu vermitteln scheinen.

3 Im Sinne ihrer aktiven Handlungsmöglichkeiten bzw. -macht im Gegensatz zum passiven oder nur re-agierenden Objekt. 
Mit dem Fortschreiten der graphischen Möglichkeiten steigt auch der Anspruch in aktuellen Spielen, wilde, unberührte Natur möglichst detailgetreu und realistisch einzubinden, wodurch diese als Raum neu erschaffen, gestaltet und kommentiert wird. Der 2018 erschienene zweite Teil des Wild-West Open World-Spiels Red Dead Redemption wurde daher auch nicht zu Unrecht in eine Tradition gestellt, die eigentlich der Literatur vorbehalten ist, nämlich das nature-writing (Hugendick, 2018). ${ }^{4}$ In der Darstellung der Natur wird in Computerspielen, analog zu anderen Medien wie Literatur und Film, ein gesellschaftlich, sozial und kulturell relevanter Kommentar zur Rezeption und Interaktion zwischen Mensch und Umwelt geleistet, der sich einerseits aus der zeitgenössischen Wahrnehmung und aus bestehenden Diskursen speist, der aber andererseits vor allen Dingen auch wiederum diese Wahrnehmung und Diskurse - und damit auch zukünftige Verhaltensweisen des Menschen in der Interaktion mit der Natur - prägt und gestaltet.

In zahlreichen Spielen setzt sich ein spätestens seit der Aufklärung existierendes Narrativ der Mensch-Natur-Interaktion fort, indem in ihrer Darstellung ein Paradoxon realisiert ist: Denn häufig fungiert die vom Menschen unberührte Natur, unabhängig davon, ob sie als Kulisse, Ressource, Gegner oder Text auftritt, als utopischer Sehnsuchts- und gleichzeitig als dystopischer Angstraum. ${ }^{5}$ Die Spielerin wird in zahlreichen Spielen in eine Simulation versetzt, in der die Natur zu weiten Teilen entweder noch unberührt erscheint (z. B. die nur von Naturvölkern oder gänzlich unbesiedelten Inselwelten zu Beginn von Wirtschaftssimulationen wie z. B. der Anno-Spielereihe ${ }^{6}$ oder Colonization - und in die Vergangenheit gesetzte Open World-Spiele wie Far Cry Primal oder die beiden Teile von Red Dead Redemption) oder aber in der die Natur sich in einer postapokalyptischen Welt den von Menschen entvölkerten Raum zurückerobert hat (wie z. B. in The Last of Us oder der FalloutReihe). Während zum einen in der Forschung bereits darauf aufmerksam gemacht wurde, dass es sich gerade bei Open World-Spielen nicht um eine "unberührte" Natur handelt, sondern dass diese durchsetzt ist "mit einem dichten und der Logik des Mediums folgenden Netzwerk aus Quests, Siedlungen, Gegnern, Landmarks und emergenten Ereignissen" (Bonner, 2018a), so wird zum anderen auch deutlich, dass das Narrativ der vermeintlich unberührten Natur unterschiedliche Facetten aufweist, die auf eine Tradition der Mensch-Natur-Dichotomie verweist und in ihrem Umgang, den das Spielprinzip von der Spielerin fordert, eine anthropozentrische,

4 Ausgehend insbesondere von Texten des 18. und 19. Jahrhunderts, insbesondere seien hier JeanJaques Rousseau und Henry David Thoreau genannt.

5 Dieses Paradoxon findet sich ebenfalls bereits bei den oben genannten Autoren Rousseau und Thoreau, aber auch im deutschsprachigen Raum in der intensiven Rezeption und Reflexion der philosophischen Auseinandersetzung um den "Naturzustand" des Menschen, so z. B. bei Christoph Martin Wieland.

6 Ausgenommen seien hierbei die Spiele Anno 2070 und 2205, die in der Zukunft spielen. 
kolonisierende und schlussendlich auch ausbeutende Strategie begünstigt - wenn nicht gar erzwingt.

Zugrunde liegt den meisten Spielen der oben bereits erwähnte Dualismus in der Darstellung und Wahrnehmung der "unberührten" Natur als locus amoenus und terribilis gleichermaßen. Einerseits erscheint sie als Wunschraum, der in der realen Welt nicht mehr vorhanden ist: "Je mehr die Natur als konkrete Lebenswelt dahinschwindet, desto mehr wird sie zu einem Sehnsuchtsraum, einem Symbol für das harmonische, gesunde Leben, einem Ort der Freiheit" (Doma, 2018, 43). Allerdings gilt dies nur für die Art von Natur, die bereits kultiviert ist oder das Potential zur Kultivierung birgt. Liboriussen bezieht sich in diesem Kontext auf eine evolutionsgeschichtliche Herangehensweise an die ästhetische Wahrnehmung der Natur, die sich auch in der Computerspielästhetik spiegle: "If we, for example, find a landscape pleasing, this positive reaction can be explained by that landscape's relatively high potential for survival" (Liboriussen, 2008, 145).

Bonner stellt die Wildnis in Computerspielen in eine Tradition von Naturparks, Landschaftsgärten und Ruinen als "kompensatorische[] Aushandlungsorte", indem "die Wildnis Erlebnis- und Erholungsort des Menschen [ist], der zugleich den Dualismus zwischen Mensch und Natur als liminalen Aushandlungsort verkörpert” (Bonner, 2018a). In Anlehnung an das Konzept von Deleuze und Guattari (Deleuze, Guattari, 2006) spricht er von "gekerbter Wildnis" bzw. "striated wilderness" (Bonner, 2018b). Der vermeintlich "glatte" Raum der unberührten Wildnis (vermeintlich, da er bereits in seiner "Gemachtheit" und im Rahmen des Mediums einer bestimmten Ordnung unterworfen ist) wird durch das Gameplay zunehmend in einen "gekerbten" überführt, also geordnet und gerastert, und damit den Bedürfnissen des Menschen im Rahmen seiner Funktion für die Spielerin - sei es als atmosphärische Kulisse, als nutzbare Ressource, als bezwingbarer Gegner oder als lesbarer Text - angepasst.

Durch das Eintreten des Avatars (in Open World-Spielen) oder den Beginn der Simulation (in Wirtschaftssimulationen) verliert die unberührte Natur ihren Status als solche, indem sie betreten, verändert und dadurch "kultiviert" wird. Denkbar wären dabei unterschiedliche Ansätze des Umgangs mit der Natur, die auch kritische Positionen und innovative Handlungsoptionen z. B. in Bezug auf den Klimawandel enthalten. Insbesondere in Mainstream-Spielen wird diese potentielle Bandbreite aber nicht ausgeschöpft (Abraham, Jayemanne, 2017, 78) - das gängige Masternarrativ des kapitalistischen "Wohlstand-durch-Wachstum"-Gedankens ist hier massiv vorherrschend $-{ }^{7}$ und dies findet zumeist auf Kosten der zu Beginn der Simulation

7 So z. B. in Landwirtschaftssimulationen, denn durch ihre utopische und unterkomplexe Darstellung, "farm games $[\ldots]$ inadvertently reprise the capitalist drama of modern agricultural history and economics" (Chang, 2012, 7). 
noch unberührten, dann aber zunehmend kultivierten bzw. bearbeiteten Natur statt: "[T] oo many games re-inscribe ideological and cultural norms that are ecologically regressive" (Smith, 2017, 117).

\section{III}

Das Bild der "gekerbten" als gerasterten Wildnis lässt sich am einleuchtendsten anhand von Wirtschaftssimulationen illustrieren und nachvollziehen. Spielreihen wie Sim City, Civilization, Anno und andere bauen seit den späten 80er und frühen 90er Jahren äußerst erfolgreich auf die Einteilung der Natur in einzeln zu betretende und zu bearbeitende Raster ('grids') auf. Während dieser Umgang mit Wildnis auch der technischen Realisierung des Spielprinzips geschuldet ist, so sollte doch nicht übersehen werden, dass damit ein Narrativ fortgeschrieben wird, das vorherrschend in der westlichen Kolonialisierungspraxis ist. Smith (2017) thematisiert dies in Anlehnung an den amerikanischen Historiker David Nye (2003), laut dem das Narrativ der USA als "zweite Schöpfung” mit vier strukturellen Veränderungen einhergeht, nämlich erstens mit der Einteilung der Landschaft in Raster, zweitens mit dem Glauben an endlos verfügbare Ressourcen, drittens mit der Ablehnung staatlicher Kontrolle und dem Vertrauen in den freien Markt sowie viertens mit der Vorstellung, dass Macht und ihre Effizienz sich ständig vergrößern (Nye, 2003; Smith, 2017). Es ist erstaunlich, in welchem Ausmaß diese Parameter auch als zugrundeliegendes Prinzip von Mainstream-Wirtschaftssimulationen $\mathrm{zu}$ beobachten sind und damit ständig wiederholt, erneuert und unkritisch übernommen werden.

Die unberührte Natur hat im Rahmen dieser Spiele nur insofern ihre Daseinsberechtigung, als sie als Folie für Technisierungs-, Expansions- und Kultivierungsphantasien für die Spielerin bereitsteht. Eine "second creation" wird aus der gottähnlichen Steuerungsperspektive top-down ermöglicht - ein eigenes Volk wird erschaffen, zähmt die Natur und schwingt sich zur Supermacht auf. Interessant ist dabei durchaus, dass die freien Handlungsmöglichkeiten der Spielerin nur eine Illusion sind - das Gros der Mainstream-Wirtschaftssimulationen gibt eine klare Erfolgsstrategie vor, die zwar individuell einige Abweichungen und Präferenzen aufweist, insgesamt aber dem Masternarrativ des westlichen und vor allen Dingen US-amerikanischen Modells, wie von Nye definiert, folgt. Im Prinzip wird die westliche Erfolgsgeschichte nacherzählt - eine denkbare Lehre aus vergangenen Ungerechtigkeiten und Fehlern z. B. im Umgang mit indigenen Völkern oder Ressourcen wird kaum gezogen. Nye $(2003,41)$ zeigt auf, wie das amerikanische Masternarrativ andere Narrative, "including those of indigenous American people, and narratives of ecological limits" (Smith, 2017, 105) unterdrückt (hat). Im Spiel 
Colonization (1994), in dem man neben einer freien Landkarte auch eine in Bezug auf die Geographie, Ressourcen und Ureinwohner halbwegs exakte Karte der Amerikas "kolonisieren" kann, wird die Ausbeutung der indigenen Völker nicht nur gebilligt, sondern geradezu gefordert. Insbesondere die Zerstörung der Inka- und Maya-Städte liefert enorme Goldschätze, die den Unabhängigkeitskrieg und damit den Spielsieg erheblich erleichtern, während eine freundschaftliche Beziehung zu den Stämmen auf Dauer kaum Vorteile birgt. Zusätzlich werden mit einzelnen "Gründervätern", die im Laufe des Spiels freigeschaltet werden, weitere Anreize zur Ausbeutung geschaffen. ${ }^{8}$

Die theoretische Chance, Natur in unberührter und noch $\mathrm{zu}$ entdeckender Form zu erleben, wird dementsprechend paradoxerweise vor allen Dingen dazu genutzt, um den Pfad (wenn auch in stark unterkomplexer Form) nachzuvollziehen, der zum Status Quo der zeitgenössischen Geschichte zurückführt. Besonders deutlich wird dies am Beispiel der Reihe Civilization, die mit dem zu beschreitenden Technologiebaum eine Genese vorschreibt, die einen "kategorische[n] Ausschluss von Entwicklungsalternativen” beinhaltet und deren "teleologisches Geschichtsverständnis einem Bedürfnis nach Sicherheit, Sinn und Fortentwicklung entgegenkommt” (Baur, 1999, 89). Während insbesondere in Bezug auf Spielreihen in der Forschung darauf hingewiesen wurde, dass die Funktion der Historizität in Computerspielen mit historischem Bezug nicht auf einer faktischen, sondern auf einer affektiven Historizität (d. h. auf Basis einer imaginierten Geschichte und ihrer ästhetischen Repräsentation) beruht, die durch die Serialität der Spiele begünstigt wird (Winnerling, 2014), so gilt dies ebenso für die Repräsentation der Natur und den Umgang mit ihr, indem sie zu einem Projektionsraum wird, der keinen Bezug mehr zur "realen" Umwelt und den mit ihr einhergehenden, komplexen Interaktionen beinhaltet.

Diese Loslösung vom "realen” Raum in Computerspielen trotz des Anspruchs an möglichst detailgetreue und graphisch ausgefeilte Repräsentation wird besonders in der unterkomplexen Darstellung von Landwirtschaftssimulationen deutlich. Chang (2012) hat ausführlich herausgearbeitet, inwiefern in solch überaus erfolgreichen Spielen wie FarmVille (2009) entscheidende Faktoren wie Wasserversorgung, Abfallwirtschaft, Ressourcenknappheit oder auch Arbeitsbedingungen vollkommen ausgeklammert werden. Auch dies hat seinen Ursprung in der Verknüpfung von einerseits bukolischpastoralen Sehnsuchtsvorstellungen und andererseits einer Technologiegläubigkeit, mit der die wilde Natur gezähmt und bequem gemacht wird, was hier laut Chang

8 So ermöglichen z. B. die Ernennung Peter Minuits und Hernan Cortes' folgende Boni: "Nachdem Peter Minuit dem Kontinentalkongress beigetreten ist, müssen Sie für von den Indianern abgetretenes Land kein Geld mehr bezahlen" und "Nachdem Cortes Ihrem Kongress beigetreten ist, werfen alle eroberten Siedlungen der Ureinwohner mehr Schätze ab, und die Schätze werden kostenlos nach Europa transportiert" (Colonization, 1994). 
bereits gar keinen Widerspruch mehr darstellt: "Pastoral in this modern form has lost any need to self-consciously examine its relation to technology, because it has already incorporated technology wholesale into its fold" (Chang, 2012, 3f.).

Die Repräsentation des Raums in einer vorindustriellen Vergangenheit oder in einer ländlichen Idylle hat damit sehr viel weniger mit historischer oder faktischer Genauigkeit zu tun als mit den Wunschprojektionen der sich in diesen Imaginationsräumen bewegenden modernen, hochtechnisierten und zumeist urbanen Gesellschaft. Die Natur wird nostalgisch verklärt und, ähnlich dem Landschaftskonzept des Englischen Gartens, in einen Raum überführt, der das Sehnsuchtsbedürfnis des Unberührten und Natürlichen der Vergangenheit mit dem Sicherheitsraum des Heimischen und Technisierten der Moderne verknüpft, ohne dass dies zwangsläufig auf den ersten Blick spürbar wäre. Allerdings existieren auch Spiele, die diesen dualistischen Mechanismus bewusst sichtbar machen - so z. B. die Wirtschaftssimulation Stardew Valley (2016), die im Retro-Look mit verpixelter

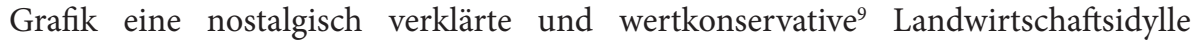
entwirft und damit auf der Metaebene einen Kommentar zum Konstruktcharakter dieses Imaginationsraums abgibt: Einerseits "geht die Pixel-Natur in Technik auf und hebt ihre programmierte Gemachtheit hervor, während [das Spiel gleichzeitig] eine verklärte Natürlichkeit in der Narration zu betonen sucht” (Schallenberg, 2018).

\section{IV}

Ein Spiel, das moderne Technik als grundsätzliches Spiel- und Narrationsprinzip vermeintlich vollkommen ausklammert, ist Far Cry Primal (2016), ein Ableger der erfolgreichen Serie, der in der mesolithischen Ära um 10.000 v. Chr. spielt. Allerdings ist nicht nur die "wilde" Natur, die einen Großteil der Open-World-Karte ausmacht, erheblich durch vorgegebene Klettervorrichtungen, Pfade und Missionen "gekerbt" (Bonner, 2018a), sondern sie wird tatsächlich auch durch die Spielerin mittels ihres Avatars mit technischen Mitteln bearbeitet und bezwingbar gemacht, deren höchst anachronistischer Charakter nur marginal hinter der Verkleidung von Pfeil und Bogen (mit der Durchschlagskraft von modernen Schusswaffen), gezähmten Tieren (die sich wie Überwachungs- und Angriffsdrohnen einsetzen lassen) oder Bienen (als Bomben) verdeckt wird. Es ist sowohl von Gaming Communities wie auch von der Forschung kritisiert worden, dass sich trotz des innovativen Ausgangspunkts das Gameplay auf zahlreichen Ebenen kaum von den anderen

9 Dieser Wertkonservativismus bezieht sich vor allem auf die Stadt-Land-Dichotomie und eine Verklärung des ,einfachen' Lebens im Einklang mit der Natur. In anderen Hinsichten bemüht sich das Spiel um Progressivität: Gleichgeschlechtliche Ehen mit der Option, Kinder zu adoptieren, sind z. B. möglich. 
Teilen der Serie unterscheidet (Majewski, 2016), wodurch das Setting und mit ihm die Darstellung der vom Menschen noch unberührten Natur kein eigenes Potential entwickelt, sondern als reproduzierbare Schablone mit rein illusorisch-ästhetischem Charakter verkommt. Es ist allerdings vor allem ein Umstand, der hier die TechnikDeterminiertheit der eigentlich technikfrei erscheinenden Umwelt besonders deutlich macht: die in Open-World-Spielen dominierende Informationstechnik, inklusive Mini-Map, Questübersicht und die durch eine gezähmte Eule (Drohne) verfügbare nachrichtendienstliche Intel. Dieser Widerspruch in sich und der damit ad absurdum geführte Anspruch, mit dem Avatar eine der mesolithischen Vergangenheit auch nur annähernd realistisch nachempfundene Erfahrung zu erleben, wird billigend in Kauf genommen, da mit ihm der potentielle Angstraum der Wildnis in einen Sehnsuchtsraum gewandelt wird, in dem das archaische Bedürfnis nach Unterwerfung der Natur bedient werden kann. Die Spielerin sieht sich hier als Urmutter eines sesshaften Stammes an den Anfängen der Schöpfung der Kultur; und dies - denn diese Illusion wird aufrecht erhalten, wenn sie auch leicht zu durchschauen ist - vermeintlich aus eigener Kraft und ohne die technischen Hilfsmittel, die dem modernen Menschen zur Verfügung stehen.

Einen sowohl in Bezug auf seine graphische Ausarbeitung, landschaftliche Differenzierung, Interaktionsmöglichkeiten und in die Natur als Text eingeschriebenen philosophischen Tiefe sehr viel komplexer gestalteten Raum bietet die Repräsentation der Natur in Red Dead Redemption I (2010) und II (2018). Die Spielmacher um Rockstar Games, die auch mit der GTA-Reihe dafür bekannt geworden sind, dass sie einerseits gängige Narrative wie den “American Dream” reproduzieren und gleichzeitig ständig kritisch kommentieren und ironisch unterlaufen, versetzen die Spielerin in den zu großen Teilen noch vorindustriellen Wilden Westen um 1900. Analog zu Far Cry Primal und anderen Open World-Spielen wird hier zu dem freien Bewegen in der "Wildnis" ein Gegenraum geschaffen in Form eines Rückzugsorts, der es dem Avatar ermöglicht, sich immer wieder in absolute Sicherheit vor wilden Tieren, aggressiven NPC oder dem Arm des Gesetzes zu retten. Dieser Raum ${ }^{10}$ ermöglicht das Heimelige im Wilden, und mit zunehmendem Ausbau auch eine Zähmung und Kultivierung des zu Beginn noch sehr einfachen und der Wildnis ausgesetzten Heims. Der "Dualismus zwischen Wildnis und Frontier" (Bonner, 2018a) wird hier in seiner bislang graphisch und spielerisch ausgefeiltesten Form zelebriert.

Das Spiel setzt sich aber trotz seiner Mainstream-Ausrichtung von zahlreichen anderen Open World-Spielen darin ab, dass die Natur in ihrer Darstellung zwar

10 Im ersten Teil ist er noch nicht so stark ausgeprägt als Zimmer auf einer Ranch mit Nebenmissionen, im zweiten Teil stellt er mit dem Bandenlager einen enorm bedeutenden und zeitintensiven Aspekt des Gameplays dar. 
einerseits glorifiziert wird, andererseits aber auch weniger romantische Seiten nicht ausgeblendet werden. Backe (2018) macht in diesem Kontext etwa auf die Reaktionen des Protagonisten beim Häuten der Tiere aufmerksam, indem dieser die widerlichen Gerüche und den unappetitlichen Vorgang kommentiert, während der Bildschirm mit Blutspritzern übersäht wird. Obwohl die Spielreihe insbesondere in Bezug auf die Nebenquests und -herausforderungen dazu einlädt, die Natur in aller Ruhe zu durchstreifen und auf mit ihr im Einklang stehende Weise zu genießen (etwa durch das Sammeln von Pflanzen), so wird die Spielerin doch an anderen Stellen dazu gezwungen, konsequent ausbeuterisch und brutal gegen sie vorzugehen, um 100\% des Spielfortschritts zu erreichen, so z. B. in der Herausforderung "Erlege einen Bären mit dem Jagdmesser und häute ihn anschließend" (Red Dead Redemption I). Ein respektvoller Umgang mit der Natur ist in dieser Aufgabe kaum möglich und in zahlreichen Spieleforen wird dazu geraten, dem Bären zunächst 3-4 mal in den Kopf zu schießen, um ihn zu schwächen, bevor man sich ihm mit dem Messer nähert. Während über Sinn oder Sinnfreiheit solcher Herausforderungen diskutiert werden kann und die Ausbeutung der Natur ebenso wie ihre Wertschätzung dem Spielkonzept inhärent sind, zwingt das Spiel doch zu einer Reflexion dieser dualistischen Interaktionsmöglichkeiten. Ein Beispiel hierfür ist die von Backe analysierte Trophäe "Manifest Destiny", ${ }^{11}$ die für die Ausrottung der Büffel (das einzige Tier im Spiel, das keinen Respawn-Effekt hat) verliehen wird und bei Erreichen öffentlich für alle Spieler der Community zu sehen ist:

Um alle, auch die optionalen, Ziele des Spiels zu erfüllen, muss eine Handlung verübt werden, die in der Spielwelt als amoralisch gegenüber der natürlichen Umwelt kodiert ist und dies auch im außerspielerischen Wertesystem der Spielerin sein sollte. Indem das Spiel dazu verführt, gegen diese Werte zu handeln, fordert es effektiv zu einer Reflexion dieser Werte, aber auch des Handelns innerhalb des Spiels insgesamt heraus (Backe, 2018, 179).

In den Red Dead Redemption-Spielen wird die Natur sowohl in ihrer Schönheit als auch in ihren brutalen Elementen, sowohl in ihrer Übermacht als auch in ihrer Verletzlichkeit differenziert dargestellt, wodurch der Handlungsspielraum der Spielerin determiniert und die Interaktion mit dem Raum reflektiert wird. Der Naturraum in Red Dead Redemption II wurde von der Forschung sogar in den Kontext von Kants Konzept des "Erhabenen" gestellt, indem die Spielerin ihm gegenüber zugleich Respekt, Bewunderung und Ehrfurcht entgegenbringe:

Sensations described as "sublime" are often associated with grand and astounding natural scenery [...]. Such phenomena were not only deemed arrestingly scenic, they also seemed to address some ancient and symbolic 
aspect of life before human influence [...]. An unmitigated confrontation with such an intense reference conveyed not only beauty, but a certain amount of awe, fear and horror as well [...]. This is reflected commonly in pre- or postapocalyptic story plots within open-world games (Roncken 2019, 6).

Eine Perspektive, die das Ambivalente des Erhabenen, die Anerkennung der schieren Macht der Natur bei gleichzeitig distanzierter Beobachtung und dem Gefühl moralischer Überlegenheit erlaubt, ist das von Bonner herausgearbeitete "prospect pacing," ${ }^{12}$ welches die Bewunderung und Abschätzung der Wildnis von einem in der Regel erhöhten und sicheren Ausgangsort bezeichnet. Von diesem Punkt aus erschließt sich auch das "concept of rural open world games not only as an interactive adaption and distillation of actuality's landscapes but also as a disclosure of the desires of post-industrial man" (Bonner, 2018b). Diese Auseinandersetzung mit dem Erhabenen in der Natur unterstreicht zwar die anthropozentrische Ausrichtung von Computerspielen und ihrer Naturrepräsentation, sie gibt aber auch Grund zur Hoffnung, dass mit dieser differenzierteren und der Natur eine Eigenständigkeit einräumenden Darstellung eine kritische und wertschätzende Perspektive ermöglicht wird, die positive Auswirkungen auf die kulturelle Wahrnehmung und Problematisierung des Klimawandels haben kann.

\section{V}

In Computerspielen stellen der Raum, seine Repräsentation und die Interaktion der Spielerin mit diesem Raum eine, wenn nicht die, das Medium entscheidende Komponente dar. Aus diesem Grunde ist es naheliegend, in Bezug auf das im Kontext des Klimawandels aktueller denn je erscheinende Verhältnis zwischen Mensch und Natur zu untersuchen, wie dieses im Computerspiel realisiert wird, welche Variationen und Handlungsmöglichkeiten das Computerspiel bietet und inwiefern hierüber Rückschlüsse auf kulturelle Wahrnehmungen der Wildnis und Wunschvorstellungen bezüglich ihrer Gestaltung gezogen werden können. Schon durch das Medium selbst erscheint in zahlreichen mit der Repräsentation von Natur beschäftigten Spielen ein scheinbares Paradox zwischen Natur und Technik, indem durch technische Realisierung von der Technik vermeintlich unberührte Natur erschaffen wird. Dieser Dualismus wird dann durch die Nutzung und Zähmung der Natur bzw. der Naturunterwerfung durch den Avatar im Spiel reproduziert und findet in der Erstellung einer sorgenfrei distanzierten und gleichzeitig aufregend immersiven Mensch-NaturInteraktion seine Auflösung. Das Gros der Mainstream-Spiele bedient sich hierbei

12 Ohne Verweis auf Bonner und ohne weitergehende soziologische oder philosophische Einbettung beschreibt Roncken das gleiche Phänomen der Perspektive für Red Dead Redemption II und stellt dabei Bezüge zu Caspar David Friedrichs Wanderer über dem Nebelmeer her (Roncken, 2019, 6). 
gängiger Narrative insbesondere westlicher Kolonialisierungsstrategien, um die Natur zu "kultivieren" und bespielbar zu machen, wobei der Natur selbst kaum oder nur sehr begrenzte Agency eigeräumt wird, indem sie zum alleinigen Projektionsraum menschlicher Machtphantasien wird. Nur wenige Spiele wagen die tatsächliche kritische Auseinandersetzung mit der Wildnis und vor allem auch mit dem negativen Einfluss des Menschen und seiner Technik auf diese. Tatsächlich scheinen jedoch gerade diejenigen Spiele, die den Widerspruch zwischen der Natur als Angst- und Sehnsuchtsraum nicht nivellieren, sondern beiden Konzepten nebeneinander Geltung einräumen und der Natur eine vom Menschen losgelöste Dynamik zusprechen, nicht nur zu den atmosphärisch gelungensten zu zählen, sondern auch zur differenziertesten Reflexion über die Interaktion mit dem Naturraum einzuladen.

\section{Bibliographie}

Aarseth, E., Allegorien des Raums. Räumlichkeit in Computerspielen, Zeitschrift für Semiotik 23.1, 2001, S. 301-318.

Abraham, B., u. a., Where are all the climate change games? Locating digital games' response to climate change, Transformation 30, 2017, S. 74-94.

Backe, H-J., Green Gaming. Vorschläge für eine Ökokritik des Computerspiel, in: Literatur und Ökologie: Neue literatur- und kulturwissenschaftliche Perspektiven (Hg. Schmitt, C. u. a.), Bielefeld 2018, S. 171-184.

Baur, S., Historie in Computerspielen. "Anno 1602 - Erschaffung einer neuen Welt", WerkstattGeschichte 23, 1999, S. 83-91.

Bonner, M., Die gekerbte Wildnis. Inszenierungen vermeintlich unberührter Natur in digitalen Spielwelten, Paidia. Zeitschrift für Computerspielforschung 2018a, http://www.paidia.de/die-gekerbte-wildnis-inszenierungen-vermeintlichunberuehrter-umwelt-in-digitalen-spielwelten/ [5. 10. 2019].

Bonner, M., On Striated Wilderness and Prospect Pacing. Rural Open World Games as Liminal Spaces of the Man-Nature Dichotomy, in: Proceedings of the 2018 DiGRA International Conference: The Game is the Message, 2018b, S. 1-18, http://www.digra.org/digital-library/publications/on-striated-wilderness-andprospect-pacing-rural-open-world-games-as-liminal-spaces-of-the-man-naturedichotomy/ [5. 10. 2019].

Chang, A. Y., Back to the Virtual Farm. Gleaning the Agriculture-Management Game, Interdisciplinary Studies in Literature and Environment 19.2, 2012, S. 237-252.

Chang, A. Y., Playing Nature. The Virtual Ecology of Game Environments, Berkeley 2013. 
Chang, A. Y. u a., Green Computer and Video Games. An Introduction, Ecozon@ 8.2, 2017, S. 1-17.

Deleuze, G., Guattari, F., 1440 - Das Glatte und das Gekerbte, in: Raumtheorie. Grundlagentexte aus Philosophie und Kulturwissenschaft (Hg. Dünne, J. u. a.), Frankfurt a. M. 2006, S. 434-445.

Doma, A., Die Natur, in: Lebenswelten - Dialoge im Deutschunterricht. Schreiben Lesen - Lernen - Lehren (Hg. Roche, J. u. a.), Tübingen 2018, S. 41-52.

Freyermuth, G. S., Games. Game Design. Game Studies. Eine Einführung, Bielefeld 2015.

Günzel, S., The Space-Image. Interactivity and Spatiality of Computer Games, in: Conference Proceedings of the Philosophy of Computer Games (Hg. Günzel, S. u. a.), Potsdam 2008, S. 170-189.

Hugendick, D., Der Himmel ist leer, Zeit Online, 25. 10. 2018, https://www.zeit.de/ digital/games/2018-10/red-dead-redemption-2-western-videospiel-rockstargames/komplettansicht [5. 10. 2019].

Liboriussen, B., The Landscape Aesthetics of Computer Games, in: Conference Proceedings of the Philosophy of Computer Games (Hg. Günzel, S. u. a.), Potsdam 2008, S. 144-155.

Majewski, J., Oldest Culture, Newest Medium. What Emerges from the Clash?, in: Proceedings of the DiGRAA 2016 National Conference, https://www.academia. edu/33180968/Oldest_Culture_Newest_Medium_What_Emerges_from_the_ Clash [6. 10. 2019].

Möring, S. u. a., Klima - Spiel - Welten: Eine medienästhetische Untersuchung der Darstellung und Funktion von Klima im Computerspiel, Paidia. Zeitschrift für Computerspielforschung 2018, http://www.paidia.de/klima-spiel-welten-einemedienaesthetische-untersuchung-der-darstellung-und-funktion-von-klima-imcomputerspiel/ [5. 10. 2019].

Nye, D., America as Second Creation. Technology and Narratives of New Beginnings, Cambridge 2003.

Roncken, P. A., Why care about virtual landscapes? Immersive Open World Games related to Positive Health, Nordes 8, 2019, S. 1-11.

Schallenberg, F., Retrovironment - "Stardew Valley" und die Naturverklärung als nostalgische Form, Paidia. Zeitschrift für Computerspielforschung 2018, http:// www.paidia.de/retrovironment-stardew-valley-und-die-naturverklaerung-alsnostalgische-form/ [5. 10. 2019].

Smith, B. u. a., Resources, Scenarios, Agency. Environmental Computer Games, Ecozon@ 8.2, 2017, S. 103-120. 
Winnerling, T., The Eternal Recurrence of All Bits. How Historicizing Video Game Series Transform Factual History into Affectice Historicity, Eludamos. Journal for Computer Game Culture 8.1, 2014, S. 151-170.

\section{Ludographie}

Black Isle Studio: Fallout (PC). Interplay 1997.

Eric Barone: Stardew Valley (Android u. a.). Chucklefish 2016.

Max Design/Sunflowers: Anno 1602 (Windows). Sunflowers 1998.

Maxis: Sim City (Amiga u. a.). Maxis 1989.

MicroProse: Sid Meyer's Civilization (Amiga u. a.). MicroProse 1991.

MicroProse: Sid Meyer's Colonization (PC u. a.). MicroProse 1994.

Naughty Dog: The Last of Us (Playstation 3). Sony Computer Entertainment 2013.

Rockstar San Diego: Red Dead Redemption (Playstation 3 u. a.). Rockstar Games 2010.

Rockstar Studios: Red Dead Redemption II (Playstation 4/Xbox One). Rockstar Games 2018.

Ubisoft Montreal: Far Cry Primal (Playstation 4 u. a.). Ubisoft 2016.

Zynga: Farm Ville (Android u. a.). Zynga 2009. 


\section{Kristina Lahl \\ Prostori strahu in hrepenenja. Reprezentacije narave $v$ računalniških igrah}

Ključne besede: računalniške igre, narava, pripoved, kultiviranje, sposobnost samostojnega odločanja

Prostor - in z njim grafična in kulturna reprezentacija okolja, interakcija $\mathrm{z}$ danim simuliranim prostorom ter navigacija $\mathrm{v}$ njem - je ključni vidik estetike in funkcionalnosti računalniških iger. Pojmu "prostora « se lahko približamo z različnimi teorijami in pristopi, ta članek pa se osredotoča na reprezentacijo narave. Analiza različnih upodobitev narave v simulacijskih in Open World igrah kaže, da je narava lahko prikazana kot locus amoenus ali kot locus terribilis, kar pa pomeni, da je instrumentalizirana. Ni upodobljena kot entiteta na sebi, temveč kot prostor, ki ga je treba raziskati, osvojiti in izkoriščati. Članek razkriva narative, na katerih temeljijo te upodobitve. Ti narativi ponavljajo vprašljivo kapitalistično dogmo, ki naravi odreka samostojnost in propagira neskončno rast človekove nadvlade. 


\section{Kristina Lahl}

\section{Spatial Narratives of Fear and Longing. Representations of Nature in Video Games}

Keywords: video games, nature, narratives, cultivation, agency

Space - and with it the graphic and cultural representation of environment, the interaction with and the navigation within a given spatial simulation - is a key aspect in the aesthetics and functions of video games. Whereas the term "space" envelops multiple different concepts and approaches, this article focuses on the representation of nature. Looking at simulation as well as open world games, different depictions of nature as a locus amoenus or respectively a locus terribilis indicate an instrumentalisation of nature not as an entity in its own right, but as a space that has to be explored, conquered and exploited. The analysis aims to uncover the underlying narratives that reiterate a questionable capitalist dogma that deprives nature of its agency while propagating endless growth and human supremacy. 